\title{
Predicting the process of industrial wastewater treatment using a hybrid intelligent model based on artificial neural network and logistic regression statistical method
}

\author{
Mirheydar Garsi EFFAT ${ }^{1}$, Jafarian AHMAD ${ }^{2, *}$ \\ ${ }^{1}$ Department of Mathematics, Urmia Branch, Islamic Azad University ,Urmia ,Iran \\ ${ }^{2}$ Department of Mathematics, Urmia Branch, Islamic Azad University ,Urmia ,Iran
}

\begin{abstract}
Today, there are different methods for treatment of wastewaters but due to their high cost and time-consuming features, an alternative precise, low cost; short-time method is always needed. Therefore, in this paper, we tried to employ a hybrid intelligent model based on artificial neural network (ANN) and logistic regression (LR) statistical method for wastewater treatment to predict the performance of malachite green removal from industrial wastewaters. Through comparing the prediction results and analyzed data, it proved that using a hybrid intelligent model based on artificial neural network and logistic regression statistical method is a valuable technique to predict the performance of malachite green removal from industrial wastewaters with high efficiency and minimum error rate.
\end{abstract}

Keywords: industrial wastewater, Green Malachite, Artificial neural network, Logistic regression, Hybrid intelligent model

\section{Introduction}

Water is one of the primary needs which is vital for human and animal lives. So due to the importance of this vital liquid in human life and water scarcity in our country, we should purify contaminated water and industrial wastewater. Industries (intentionally or not) cause the entrance of pollutants to water resources. So protection of the environment and attention to its condition are of important concern of today human society.

Dyes, heavy metals, and other pollutants resulted from industrial wastewaters are the major part of organic compounds which pollute natural waters. Therefore, removal of these pollutants from wastewaters is essential $[1,2]$.

\footnotetext{
* Corresponding auther

Email: jafarian5594@yahoo.com
} 
Textile wastewaters cause the entrance of toxic dyes to water cycle system. These dyes are unrenewable and resistant to ordinary optimum decomposition processes [3,4]

The stated problems have led to great efforts in the removal of pollutants and there are so many proposed methods for removal of pollutants from water resources which include physical, physical-chemical, chemical and biological methods to treat wastewaters. One of the effective methods to remove pollutants from wastewaters is using Polyaniline /wheat husk ash nanocomposite.

Employing methods for combining nanoparticles and other substances (especially polymers) and using them as nanocomposites is a great step to accelerate and increase the efficiency of pollutants removal.

Ghanbary et al (2012) modeled the removal of malachite green by using polyaniline nanocomposite coated on rice husk (PANI/RHA) and comparing its catalytic activity with Titanium dioxide nanoparticles $\left(\mathrm{Ag} / \mathrm{TiO}_{2}\right)$. The studies showed that Titanium dioxide nanoparticles are better in the removal of malachite green compared to PAN/RHA [5].

Jafarian et al (2011) modeled the malachite green removal in the presence of copperpolyaniline from textile wastewaters using the hybrid neural network and metaheuristic algorithms [6]. Jinquan Wan et al (2011) predicted the quality of wastewater come from paper mill using adaptive-network-based fuzzy inference system [7]. Pommier et al (2007) predicted the influence of water on solid wastes in landfill site using logistic regression statistical model [8].Michael et al (2014) used the solar energy to treat olive mill wastewater using a pilot-scale photocatalytic reactor after coagulation- flocculation [9]. Han Honggui et al (2014) presented an approach for online fault detection in wastewater treatment process using fuzzy neural network [10]. Ghanbari et al (2015) modeled the separation of malachite green in the presence of polyaniline/wheat husk ash nanocomposite (PANI/WHA) by using the artificial neural network and also experimental method. With this model, polyaniline/wheat husk Ash nanocomposite preparation and the efficiency of malachite green removal has been studied by using PANI/WHA in different conditions affected by various factors such as volume of PANI/WHA, initial concentration of MG, UV intensity and the irradiation time and they concluded that using neural network approach is more efficient than experimental one [11]. Therefore, using experimental approaches reduce reliability due to particular complications and high-cost. Different present approaches to remove pollutants aren't really efficient due to the broadness of conditions, so the techniques based on experimental data and their progress for predicting the results of processes should be clear and accurate. 
Therefore in this paper, we used a hybrid intelligent model based on artificial neural network and logistic regression statistical method which is a powerful tool in modeling complicated systems.

ANN is a simple model of the biological nervous system and for the first time in 1943, it was created by McCulloch and Pitts by employing some simple neurons and had remarkable computational power [12].

Artificial neural networks are one of the accomplishments that can investigate complicated and unknown phenomena by simulating the brain and received so many attentions due to special features such as high-speed computational ability, trainability and generalizability, resistance to change of parameters, and low-cost. ANN has suitable flexibility but increases the number of variables, the number of neurons and number of layers in the artificial neural network could complicate its structure and lead to its unsustainability [[13]. On the other hand, in some case, variables are highly correlated and if we use all of them, it can cause repetitive entering [14] which prevents us from finding the optimal models. Therefore, with regards to water scarcity, we used a hybrid ANN-LR model which is an approach based on a hybrid structure of multi-layer Perceptron (MLP) Neural networks and logistic regression statistical model, to estimate the efficiency of Malachite green removal in industrial wastewaters for avoiding contamination of waters and protection of the environment. In this study, at first we used ANN to directly predict the removal of MG pollutants from wastewaters and next we did this by LR statistical model and then we investigated by Hybrid ANN-LR model. Finally, we evaluated the accuracy of these models in predicting the industrial wastewater treatment process.

\section{Materials and methods}

\subsection{Artificial neural network}

In general, a biological neural network is a series of interconnected neurons. Each neuron could connect to so many neurons and the total number of neurons and their connections could be a lot. These connections which also called synapses are composed of axons and dendrites. The signals are passed by a synapse located on the membrane of the neuron. Fig.1 shows a nerve [12]. As can be seen, the neural network has a continuous structure in parallel forms. Artificial neural network is a process data system. A data structured designed by programming could work like a neuron and by creating a network of 
neurons and employing a learning algorithm, we could train it. Each neuron could be activated when its received signals exceed a specified threshold.

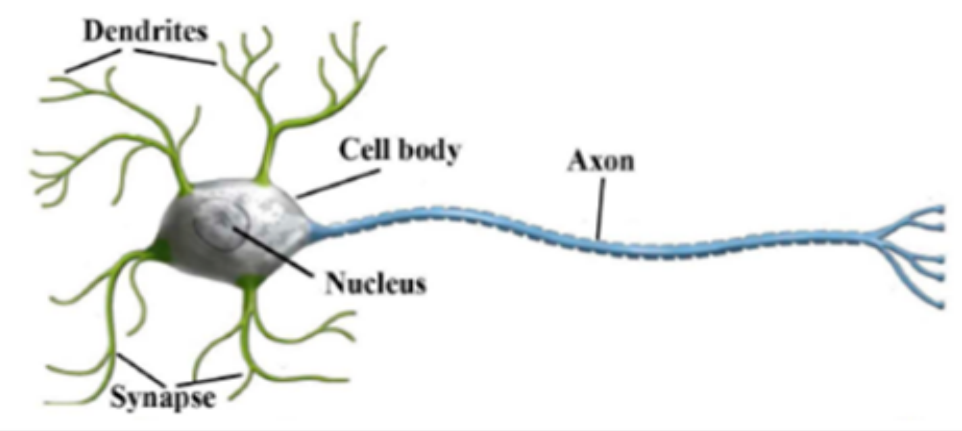

Fig. 1: A nerve

2.1.1. The structure of artificial neural network

A neural network consists of layers and weights. The behavior of network depends on the relationships among members. In general, there are three neuron layers in neural networks:

- Input layer: Receiving raw information that was fed into network

- Hidden layer: These layers process the data. There could be more than one layer [15]

- Output layer: The performance output layer depends on the performance of hidden layer and relationship of hidden and output layer (Fig.2).

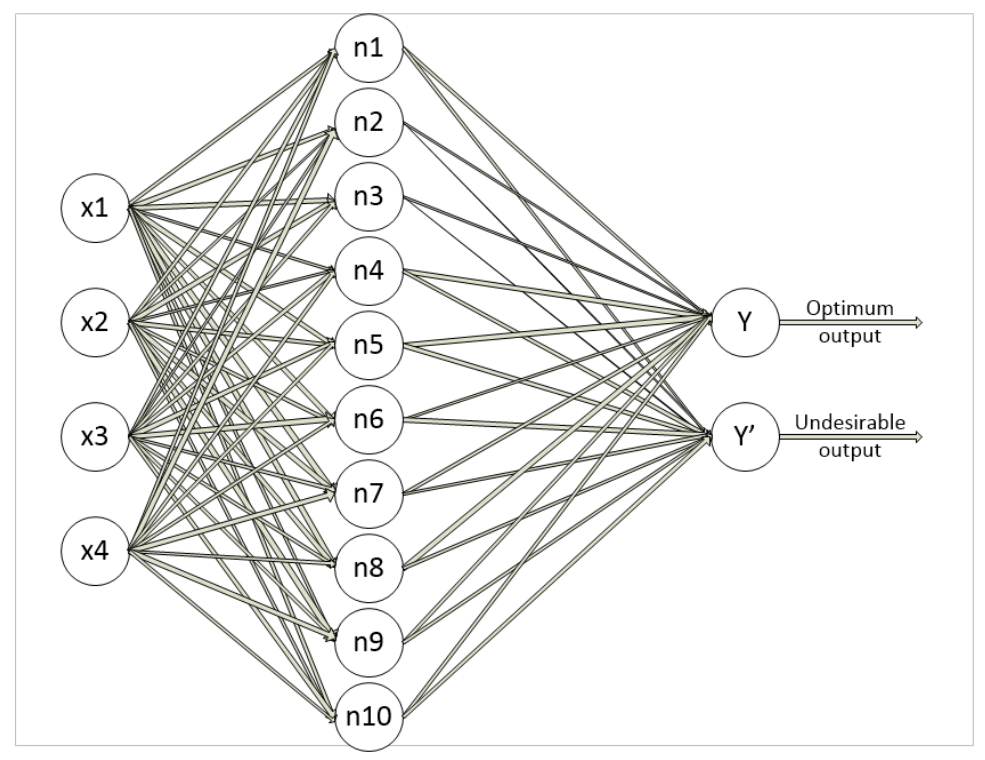

Fig. 2: Schematic of a two-layer neural network with one hidden layer

In this networks, the neurons of each layer have specific roles. The connections between neurons have weights associated with them. Usually, the inputs came from outside of network are applied to neurons of one layer. These inputs are added to weights and then sent to 
activation function of each neuron. Then these applied inputs are processed and output of the neuron is sent to next layers and finally, output layer which is the answer of the network to inputs. Output function depends on a constant value called bias. Output vector is as follows:

$n=W * P+b$

$a_{\text {out }}=f(n)$

where $\mathrm{P}$ is input vector, $\mathrm{a}_{\text {out }}$ is output vector, $\mathrm{f}$ is activation function, $\mathrm{b}$ is the bias value and $\mathrm{W}$ is weight matrix. The activation function is chosen on problem requirements and in practice, the number of these chosen functions are limited.

\subsubsection{Multi-layer Perceptron (MLP)}

One of the mostly used neural networks is Perceptron. This kind of networks can do a precise non-linear mapping by choosing the appropriate number of layers and neurons. This network consists of three layers (input, hidden, output). The number of neurons in input and output layers are determined by requirements of the problem. However, the number of neurons in hidden layers and also the number of these layers are determined by trial and error according to designer's opinion to minimize the error.

This network learns based on error back-propagation algorithm. Real outputs are compared to desired outputs and weights are determined and supervised by back-propagation algorithm till finding the suitable pattern.

For input pattern $\mathrm{p}$, the square of output error for all the neurons of output layer is as follows [16]:

$E_{p}=\frac{1}{2}\left(d^{p}-y^{p}\right)^{2}=\frac{1}{2} \sum_{j=1}^{p}\left(d_{j}^{p}-y_{j}^{p}\right)^{2}$

where $\mathrm{d}_{\mathrm{j}}^{\mathrm{p}}$ is desired out for the $\mathrm{jth}$ neuron in output layer, $y_{j}^{p}$ is real output for the $\mathrm{jth}$ neuron in output layer, $S$ is the dimensions of the output vector, $y^{\mathbf{p}}$ is real output vector and $d^{p}$ is desired output vector. Total error $\mathrm{E}$ for pattern $\mathrm{p}$ is:

$E=\sum_{p=1}^{p} E_{p}=\frac{1}{2} \sum_{p=1}^{p} \sum_{j=1}^{z}\left(a_{j}^{p}-y_{j}^{p}\right)^{2}$

To minimize the cost function E, weights are changed by gradient descent algorithm. The learning process stops when the total error (E) for pattern $\mathrm{p}$ is less than a specified threshold or the number of learning periods is reached [16]. 


\subsection{Logistic regression statistical model}

Logistic regression is a kind of regression where independent variables can be in terms of quantified or nominal scale but dependent variable is a two-level nominal variable. Logistic regression is a statistical method belonged to the generalized linear statistical method. It can predict the occurrence of an event by using independent variables. The important point in regression is that dependent variable is a two-level variable where 0 means the event isn't happening and 1 means the event is happening [17].

This model of regression looks like regular regression with only this difference that the estimation method of coefficients aren't the same and rather minimizing squared errors (like regular regression), it maximize the occurrence probability of an event. Logistic regression uses chi-square and Wald statistics. Logistic regression is like linear regression except for dependent variable. In logistic regression, there's a term for the dependent variable which is odds. Odds of an event reflects the probability that the event will take place. Probability is between 0 and 1 but odds could be more than 1 . The keyword in logistic regression is logit function which gives the logarithm of odds.

Logistic regression has two properties. First, conditional distribution y|x which is a Bernoulli distribution instead of a Gaussian distribution because the dependent variable is a two-level kind. Second, predicted value are probabilities between 0 and 1 which are obtained by the logistic distribution function. Therefore, logistic regression predicts the output probability. In this study, the purpose of logistic regression is to find the best model to describe the existed relations between a set of independent variables including PANI/WHA volume, MG concentration, UV intensity and irradiation time and one dependent variable which is a nominal variable describing presence of contamination (or not) in industrial wastewaters.

In this research, 1 is considered for $70 \%$ removal of MG from industrial wastewaters as a desirable output and 0 is considered for less than $70 \%$ removal of $\mathrm{MG}$ as undesirable output. The general of binominal logistic regression equation is as follows:

$\log i t(p)=\ln \left(\frac{p}{1-p}\right)=\alpha+\beta_{1} x_{1 i}+\cdots+\beta_{k} x_{k i} \quad i=1,2, \ldots, n$

where:

P: probability of occurrence (here, probability of contamination)

$x_{i}(i=1,2, \ldots \ldots, n)$ independent variable

$B_{i}(\mathrm{i}=1,2, \ldots . ., \mathrm{n}):$ The determined coefficients for model by regression

$p=\operatorname{Pr}\left(Y_{\mathrm{i}}=1\right)$ 
Bulletin de la Société Royale des Sciences de Liège, Vol. 85, 2016, p. 304 - 320

$p=\operatorname{Pr}\left(Y_{\mathrm{i}}=1 \mid X\right)=\frac{e^{\alpha+\beta_{1} x_{1, i}+\cdots+\beta_{k} x_{k, i}}}{1+e^{\alpha+\beta_{1} x_{1, i}+\cdots+\beta_{k} x_{k, i}}}$

\subsection{The proposed method (Hybrid artificial neural network and logistic regression)}

In this paper, at first, we presented an introduction of artificial neural network model and logistic regression statistical model to predict industrial wastewater treatment and then we provided an accurate prediction of wastewater treatment by hybridizing neural network and logistic regression. Firstly, necessary lab parameters were introduced (Table 1). Next, we applied logistic regression and statistical analysis on available parameters and determined the effect of each of them on outputs of data and output probability for each of rules (neural network inputs). The proposed method presents more accurate prediction with less error.

Then, we applied the results of logistic regression on neural network and its effect on neural network learning was great. We discussed the results of neural network and hybridizing them with logistic regression in results and discussion section.

\subsubsection{Hybrid neural network model}

We used multi-layer perceptron as an ANN model. It learns by supervised backpropagation gradient descent algorithm (Rumelhart, McClelland, and PDP group 1986). In general, we have two purposes of generating hybrid models:

1. Using statistical model to choose input variables for neural network model

2. Using output as an estimated probability for input of neural network model If we have too many variables or the number of neurons or layers in the neural network are high, the neural network may encounter some problems due to spending too much time to choose and process variables. Therefore, we could reduce its problems as follows:

1. Using statistical models to preselect variables could decrease the risk of choosing too many variables and the time spent for choosing

2. Using outputs of a statistical model as inputs of an artificial neural network could effectively compress information and let the potential variables work

You can see a schematic of the hybrid neural network in Fig. 3. As can be seen, the steps are like this:

Step 1: The inputs $x_{i}$ along with output y (target) enter LR statistical model

Step 2: LR model processes the data and information (table 2) along with estimated coefficients B are obtained 
Step 3: The estimated coefficients along with inputs $\left(\mathrm{x}_{\mathrm{i}}\right)$ enter Mnrval function and outputs are determined as desirable or undesirable probabilities.

Step 4: Inputs and outputs of LR model enter the neural network and the output of hybrid network is the final answer.

Therefore, we use the processed data of LR for hybrid ANN-LR model and the output of statistical model is the input of the neural network.

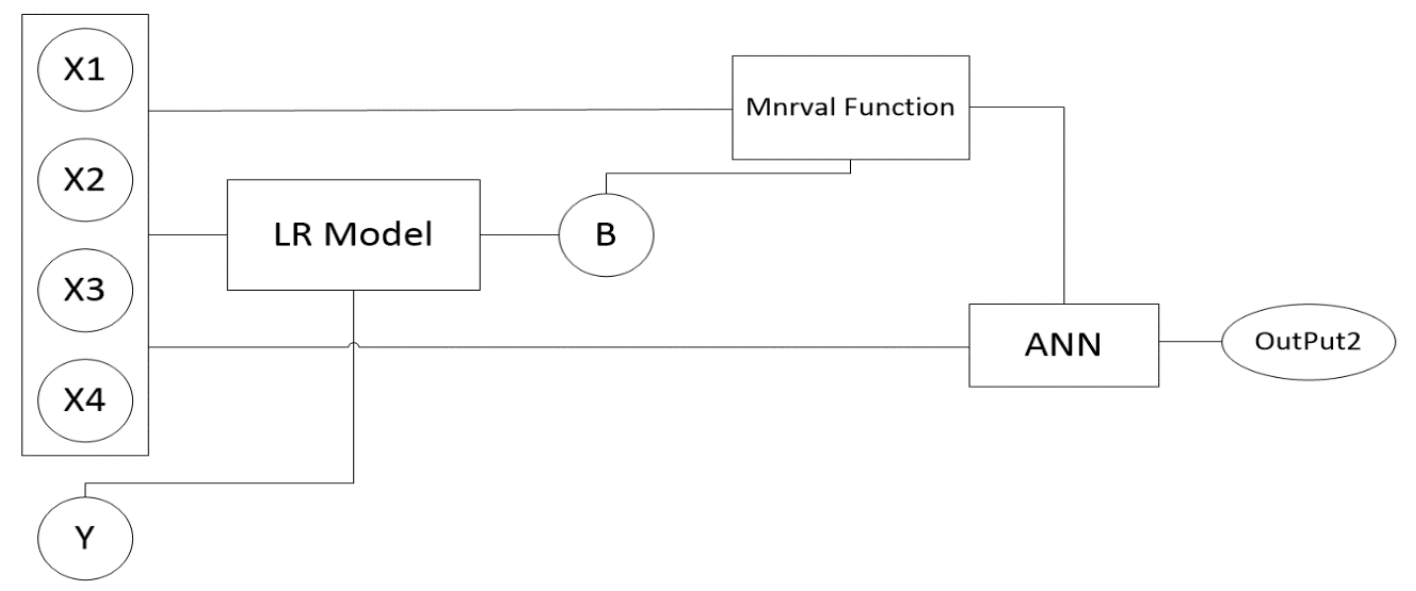

Fig. 3: Schematic of hybrid neural network

\section{Results and discussion}

\subsection{Data preparation}

We experimentally investigated the prediction of malachite green removal from industrial wastewaters using polyaniline/wheat husk ash nanocomposite after several tests on available data [11].

We studied this nanocomposite under X-radiation (XRD) and scanning electron microscope (MSE) and separating MG from wastewaters depends on the initial dosage of PANI/WHA, the initial concentration of malachite green and UV intensity and irradiation time.

The four parameters of the initial dosage of PANI/WHA, the initial concentration of malachite green and UV intensity and irradiation time are considered as input variables and removal of malachite green in percent considered as the output variable. Variables are showed in Table 1. 
Table 1: Variables of study

\begin{tabular}{cc}
\hline VARIABLE & RANGE \\
\hline Input Layer & $\mathbf{0 . 1}-\mathbf{1 . 5}$ \\
$x_{1}=P A N L / W H A$ initial dosage $(\mathrm{g} / \mathrm{L})$ & $\mathbf{1 - 1 2}$ \\
$x_{2}=M G$ initial concentration $(\mathrm{mg} / \mathrm{L})$ & $\mathbf{8 . 3 - 4 0 . 6}$ \\
$x_{3}=U V \quad$ light intensity $\left(\mathrm{W} / \mathrm{m}^{2}\right)$ & $\mathbf{0 - 2 0 0}$ \\
$x_{4}=$ Irradiation time $(\mathrm{min})$ & \\
Output Layer & $\mathbf{0 - 1 0 0}$ \\
Y=Removal of $\mathrm{MG}(\%)$ & \\
\hline
\end{tabular}

\subsection{The results of artificial neural network}

The performance of MG removal was predicted by using PANI/WHA nanocomposite and ANN model which had three layers (40 neurons in layer one, 14 neurons in layer two and one neuron in the last layer). In this research, we classified input data randomly in three categories from collected statistical data:

a. $70 \%$ of data goes in training data

b. $10 \%$ of data goes in validation data in case of network overfitting.

c. $20 \%$ of data goes $\mathrm{n}$ test data to evaluate the efficiency of final network

In this research, different numbers of neurons ( 5 to 40 ) were used in the hidden layer and 1 neuron was used in the output layer. The maximum number of repeats is 500 and threshold error is $10^{-6}$. Also, we used mean square errors (MSE) and root mean square total (RMST) as operating error and we evaluated the efficiency of network by below relation:

$M S E=\frac{1}{N} \sum_{j=1}^{N}\left(A_{j}-Y_{j}\right)^{2}$

where $\boldsymbol{A}_{\mathrm{j}}$ and $\boldsymbol{Y}_{\mathrm{j}}$ are evaluated operating index and predicted value, respectively and $\mathrm{N}$ is the total number of data.

We used trainlm function to train the network. To do this, we generated a feedforward network and by calling the newff function, we generated a three-layer network with tansig transfer function in hidden layers $(1 \& 2)$ and purelin linear transfer function in the output layer. We used regression coefficient and mean squared error (MSE) to evaluate the 
efficiency and accuracy of this model. All computations are done by 2013b.MATLAB 8.2 software using toolbar ANN. The Stop of learning procedure happened at epoch 155. The diagram of learning errors is plotted by clicking on performance key in learning window (Fig. 4).As can be seen, the mean squared error of network is high but it gradually decrease. The diagram includes three error curves which train data is blue, test data is red and validation data is green and curves shows that all these errors have the same behavior and properties and best efficiency was obtained at epoch 135 with 0.044 mean squared error.

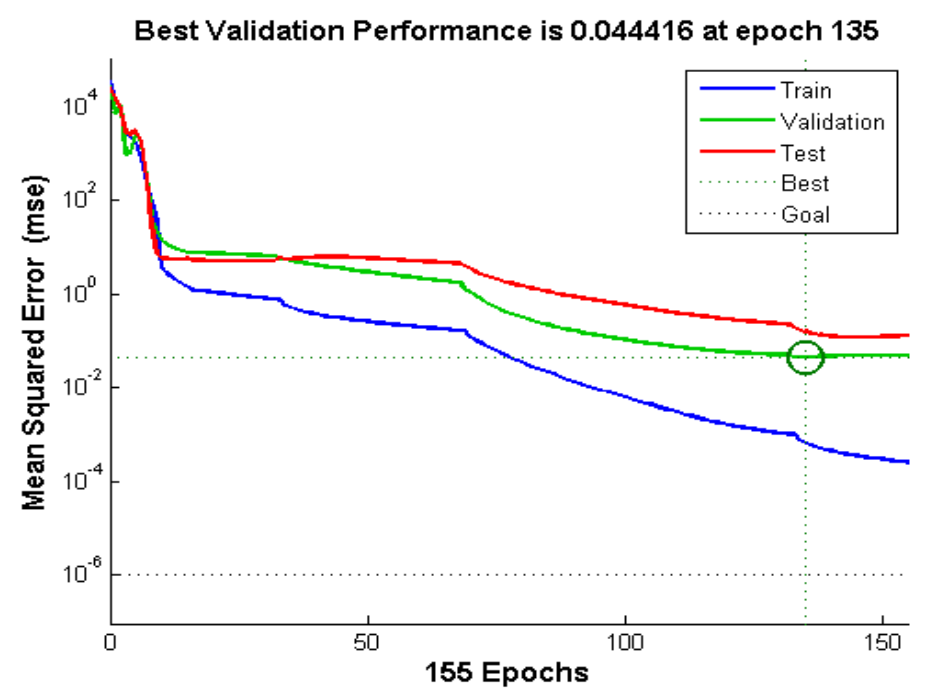

Fig. 4: The diagram of neural network performance (mean squared error)

We analyzed the reaction of network for MG removal from wastewater using PANI/WHA for the three datasets (training, validation, and test) by clicking on Regression key in learning window (Fig. 5).

As can be seen, the outputs of training, validation, and test sets reconcile on target (Regression coefficient $=1$ ) and it shows good relations between the results of all three datasets. 

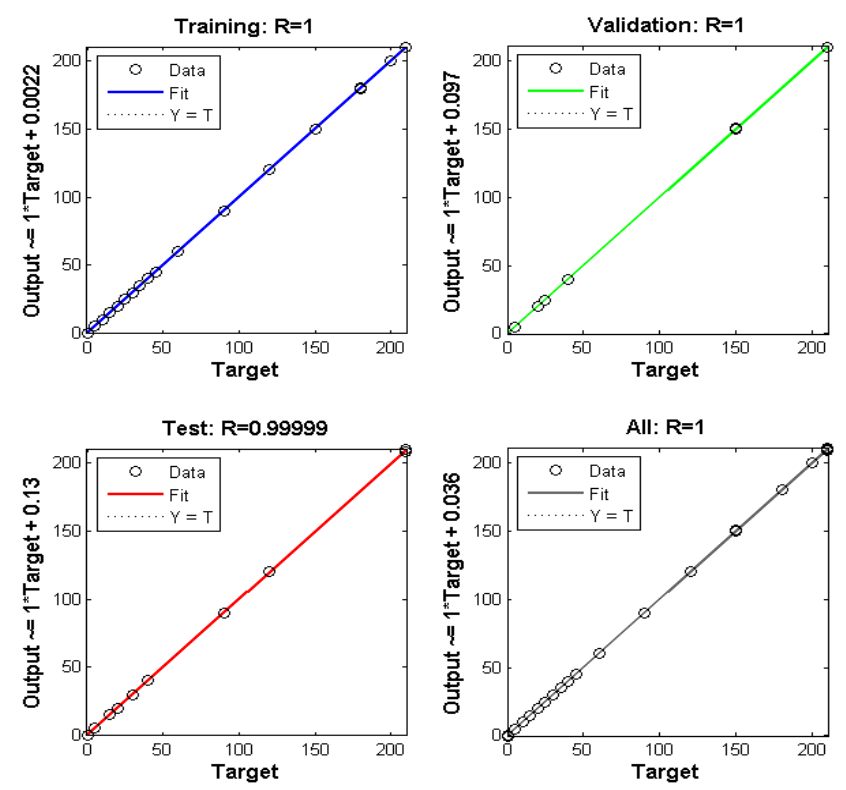

Fig. 5: The regression diagrams of network

Fig.6 shows the histogram of neural network performance to remove malachite green for three datasets (training, validation, and test) and most of the errors are between - 0.02036 and 0.06712 .

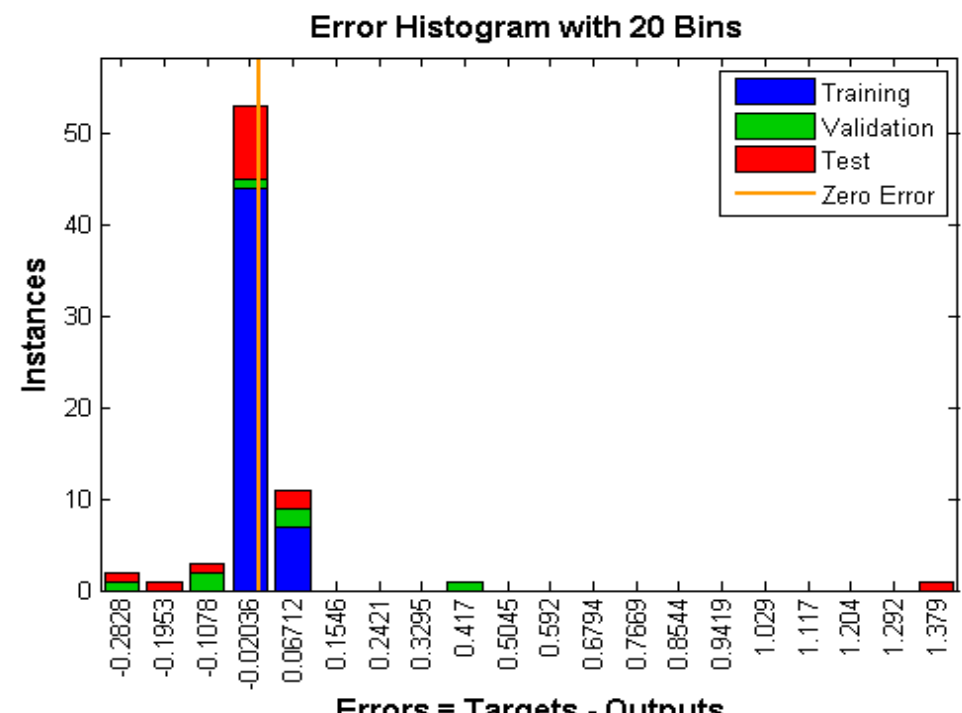

Fig. 6: Histogram of resulted errors from simple artificial neural network

\subsection{The results of regression}

The purpose of this study is to find the probability of contamination presence/non-presence in the treated industrial wastewaters. Therefore, we use logistic regression to model treatment. 
This study is a correlational one. We used feedforward method for the fitting of logistic regression and significance of regression coefficients were measured by maximum likelihood function and Wald test. The likelihood function is a method to measure the significance of regression coefficients. Maximum likelihood compares the observed values to the predicted ones and Wald test is one of the methods to measure the significance of logistic regression coefficients.

In table 2, you can see the variables along with estimated coefficients (B), the significance level of input variables (p-value), the significance level of statistical test (t-test) and standard error (SE) of the usable output of treated wastewater to unusable output.

Table 2: Estimated coefficients and usable output probability of treated wastewater to unusable output probability

\begin{tabular}{|c|c|c|c|c|}
\hline Variable & $\mathbf{B}$ & SE & p-value & t-value \\
\hline $\begin{array}{c}x_{1}=\text { PANI } / \text { WHA initial } \\
\text { dosage }(\mathrm{g} / \mathrm{L})\end{array}$ & 6.9813 & 2.6606 & 0.0087 & 2.6239 \\
\hline $\begin{array}{c}x_{2}=M G \text { initial oncentration } \\
(\mathrm{mg} / \mathrm{L})\end{array}$ & -3.0221 & 76.6987 & 0.9686 & -0.0394 \\
\hline $\begin{array}{c}x_{1}=U V \text { light intensity } \\
\left(\mathrm{W} / \mathrm{m}^{2}\right)\end{array}$ & 0.0344 & 0.02302 & 0.1352 & 1.4937 \\
\hline$x_{4}=$ Irradiation time $(\min )$ & 0.0250 & 0.0079 & 0.0015 & 3.1779 \\
\hline
\end{tabular}

According to table(2) logistic regression equation is as follows:

$\mathrm{y}=6.9813 \mathrm{x}_{1}-3.0221 \mathrm{x}_{2}+0.0344 \mathrm{X}_{3}+0.0250 \mathrm{x}_{4}$

Fig. 7 shows the diagram of estimated coefficients of the usable output of treated wastewater versus the unusable output. In Fig. 8, the horizontal axis is available parameters (4 parameters of table 1) and the vertical axis shows their significance level usable output to unusable output of treated wastewater.

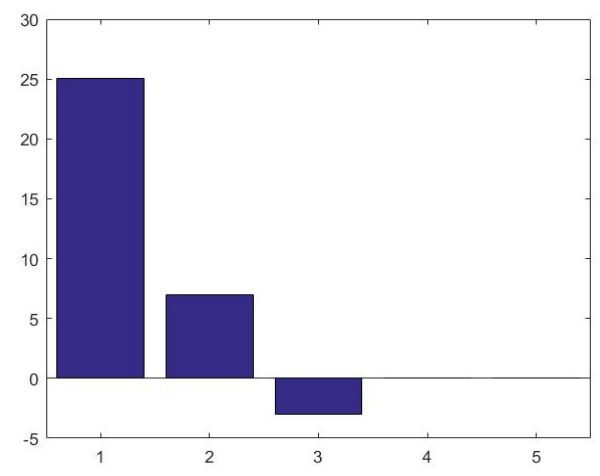

Fig.7: Diagram of estimated coefficients 


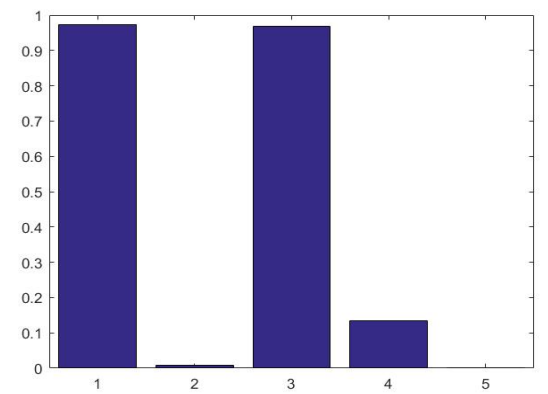

Fig. 8: The significance level of available parameters

\subsection{The results of proposed method}

We applied the inputs of table 1 to hybrid neural network and logistic regression by using MATLAB software and the below diagrams were achieved. Fig.9 shows the error decrease by the number of epochs and sum of squared error. As can be seen, mean squared error is small and best performance is achieved at epoch 18 and it is apparently better compared to Fig. 4 and hybrid model has less error than the simple neural network.

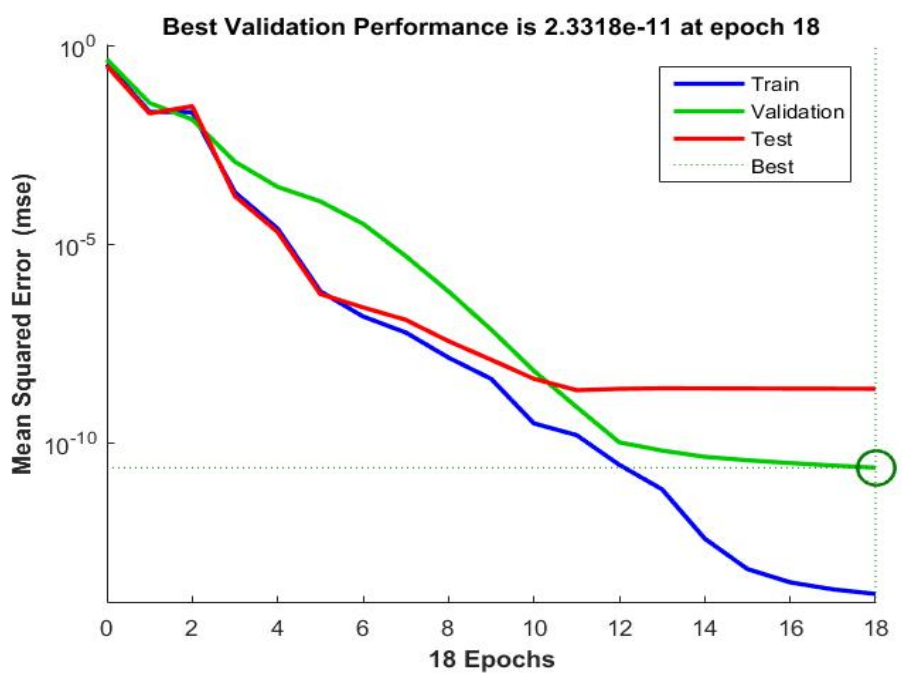

Fig. 9: The performance of hybrid neural network with logistic regression

Fig. 10 shows the regression diagram of hybrid neural network with logistic regression model for training, validation, test, and total data. As can be seen, outputs of training, validation, and test dataset are reconciled perfectly on target vectors and also diagrams of Fig. 10 have better performance compared to diagrams of Fig. 5. 

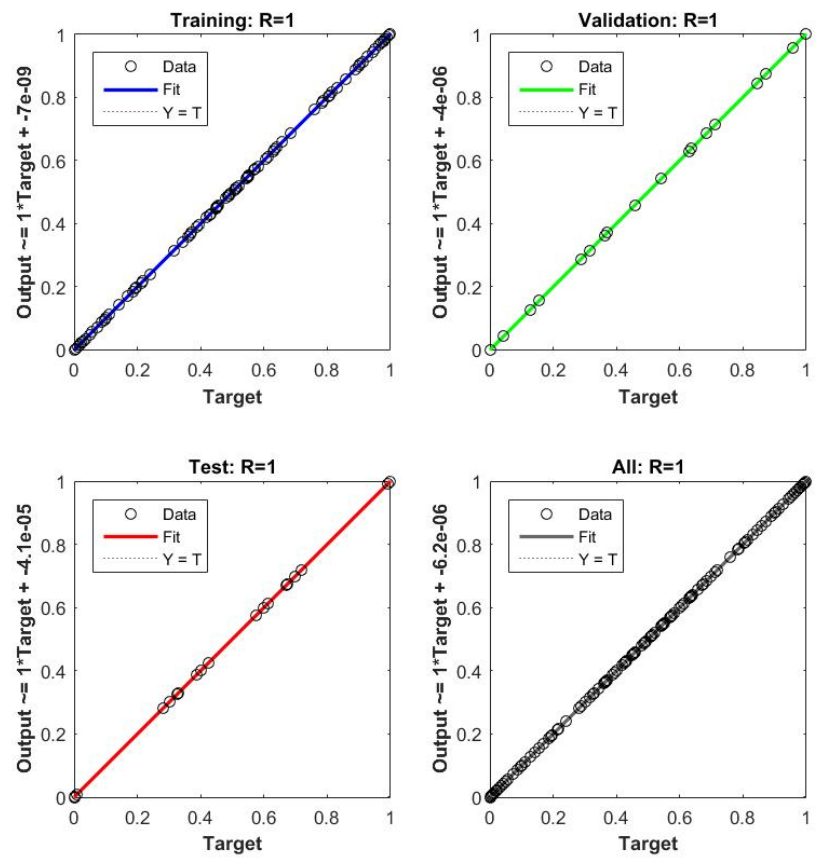

Fig. 10: The obtained regressions of hybrid neural network with logistic regression model

Fig. 11 shows the histogram of the value errors of the hybrid neural network with logistic regression model as a bar chart. The bar blue shows training data, green is validation data and red bar shows the test data. As can be seen, most of the errors are between $-7.5 \times 10^{-6}$ and $7.53 \times 10^{-6}$, however two error points of test data are at -0.00014 and 0.000143 and two error point of validation data are at $2.26 \times 10^{-5}$ and $-2.3 \times 10^{-5}$ which shows the small error of prediction compared to histogram of simple neural network.

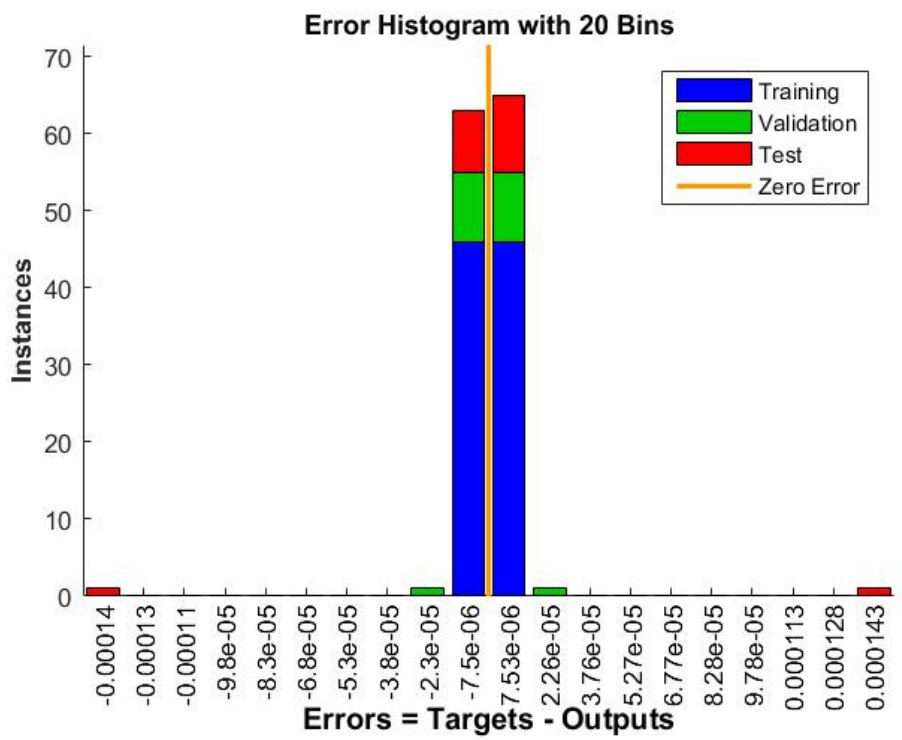

Fig. 11: Error histogram of hybrid neural network with logistic regression 


\section{Conclusion}

The main purpose of this paper was comparing the accuracy of three different models (Artificial neural network, Logistic regression, and hybrid ANN-LR) for predicting the percentage of malachite green removal from industrial wastewaters, so we can choose one method as optimum.

Independent variables in all three models included: initial dosage of polyaniline/wheat husk ash (PANI/WHA) and initial concentration of malachite green and UV light intensity and irradiation time and dependent variable in neural network was the percentage of MG removal and in logistic regression was the output probability of MG removal (\%) (More than 70\% desirable and less than $70 \%$ undesirable). In the hybrid intelligent model, this variable was considered as the probability of desirable or undesirable treated wastewater.

In this paper, we determined the significance level of each variable by logistic regression and the most influential factor on the prediction of MG removal i.e. initial concentration of $\mathrm{MG}$ was predicted. Also, we predicted the performance of MG removal by PANI/WHA nanocomposite by using ANN model which was applied in a three-layer network (40 neurons in layer one, 14 neurons in layer two and one neuron in the last layer) and error backpropagation algorithm.

With regards to large numbers of neuron and layer in the model, the network is really complicated. However, in the hybrid model, the less number of neurons (10) and two output reduced the complexity and increased the efficiency. Therefore, we could spend less time choosing and processing variables by using hybrid models.

To evaluate the performance of these models, we can see that in achieved diagrams in Fig. 4 and Fig. 9, the error for neural network is 0.04 and for hybrid model is $2.3 \times 10^{-11}$.

So, with regards to results of comparing models, we can see that hybrid neural network is much better than simple neural network or logistic regression statistical model. This difference could be related to the ability of hybrid model in identifying the mutual influence of variables on each other and available patterns among data, less error, and more accuracy of this model. Also, we could resolve most problems of wastewater treatment plants such as lack of experts in plants, inability to predict the condition input and output wastewater by using the hybrid model. The hybrid model is advanced technology and optimizing method to increase the accuracy of intelligent prediction of industrial wastewater treatment processes. Also, this model could reduce the high cost of wastewater treatment that different industries have to pay. 


\section{References}

[1] Modirshahla N., Behnajady M.A. and Ghanbary F.(2006), Decolorization and mineralization of c.l. Acid Yellow 23 by Fenton and photo-Fenton processes. Dyes pigmd ents, 2007;73(3): 305-310.DOI 10.1016/j.dyepig.01.002.

[2] Behnajady M.A, Modirshahla N. and Ghanbary F.(2007), A kinetic model for the decolorization of C.I. Acid Yellow 23 by Fenton process. J. Hazard. Mater., 2007; 148(1-2):98-102.DOI 10.1016/j. jhazmat..02.003.

[3] Muruganandham , M., Swaminathan M .(2004), Photochemical oxidation of reactive azo dye with UV/H2O2 process . Dyes Pigments ., 62: 269-275. DOI 10.1016/j. dyepig.2003.12.006.

[4] Daneshvar N., Rabbani M., Modirshahla N. and Behnajady M.A.(2005), Photooxidative degradation of Acid Red 27 in a tubular continuous-flow photoreactor: influence of operational parameters and mineralization products.J Hazard. Mater., 118: 155- 160. DOI 10.1016/j.jhazmat.2004.10.007.

[5] Ghanbary F., Modirshahla N., Khosravi M., Behnajady MA.(2012), Synthesis of TiO2nanoparticles in different thermal conditions and modeling its photocatalytic activity with artificial neural network, J. Environmental Sciences., 24(4) 750-756.

[6] Jafarian, A., Ghanbary, F.(2011), Using fuzzy interpolation for studying removal of Tartrazin by UV/TiO2 process, J. of Basic and Applied Sciences, 5(11), PP. 2230-2236.

[7] Wan, Jinquan, et al.( 2011). "Prediction of effluent quality of a paper mill wastewater treatment using an adaptive network-based fuzzy inference system." Applied Soft Computing , 11.3 : 3238-3246.

[8] Pommier, S., et al.(2007), "A logistic model for the prediction of the influence of water on the solid waste methanization in landfills." Biotechnology and bioengineering 97.3 : 473-482.

[9] Michael, I., et al. (2014), "Utilizing solar energy for the purification of olive mill wastewater using a pilot-scale photocatalytic reactor after coagulation-flocculation." Water research $60: 28-40$.

[10] Honggui, Ha, Li Ying, and Qiao Junfei.(2014), "A fuzzy neural network approach for online fault detection in waste water treatment process." Computers \& Electrical Engineering 40.7 : 22162226.

[11] Ghanbary, F., Jafarian , A.(2015), Polyaniline/wheat Husk Ash Nanocomposite Preparation and Modeling Its Removal Activity with an Artificial Neural Network . Chiang Mai Journal of Science., 42(3):1-12.

[12] Zurada, J.M .(1992), Introduction to Artificial Neural Systems, West Publishing Copany, New York.

[13] Haykin, S.(1999), Neural networks:a comprehensive foundation, 2nd Ed., Prentice Hall., New Jersey, USA 
Bulletin de la Société Royale des Sciences de Liège, Vol. 85, 2016, p. 304 - 320

[14] Zhang, Y.X.( 2007), Artificial neural networks based on principal component analysis input selection for clinical pattern recognition analysis. Talanta. 73 (1):68-75.

[15] Daneshvar N., Khataee A.R. and Djafarzadeh N.(2006), The use of artificial neural networks (ANN) for modeling of decolorization of textile dye solution containing C. I. Basic Yellow 28 by electrocoagulation process. Journal of Hazardous Materials B, 2006; 137(3): 1788-1795. DOI 10.1016/j. jhazmat..05.042.

[16] Simon Haykin. (1999), “ Neural Networks”. Macmillan College Publishing Company.

[17] Garcia-Rodriguez, M., J.A. Malpica, B. Benito and M. Diaz.( 2008), Susceptibility

assessment of earthquake-trigged landslides in El Salvador using logistic regression.Geomorhpology, 95: 172-191 